\title{
Secondary Frequency Automatic Control Method for Micro-grid Inverters
}

\author{
Jiajian HUANG \\ Electric Power Research Institute of Guangdong Power \\ Grid Co., Ltd \\ Guangzhou, Guangdong Province, China \\ E-mail: huangjiajian@gddky.csg.cn
}

Xin LI

Electric Power Research Institute of Guangdong Power Grid Co., Ltd

Guangzhou, Guangdong Province, China

E-mail: lixin@gddky.csg.cn

\author{
Xiaogang XU \\ Electric Power Research Institute of Guangdong Power \\ Grid Co., Ltd \\ Guangzhou, Guangdong Province, China \\ E-mail: xuxiaogang@gddky.csg.cn
}

\author{
Chaoming WANG* \\ Nanjing Soft Core Co., Ltd \\ Nanjing, Jiangsu Province, China \\ E-mail: 77065131@qq.com \\ +* Corresponding author
}

\begin{abstract}
Micro-grid is as an effective technology for controlling the influx of distributed generations and improving the percentage of renewable energy generation, both of which are researched all over the world. The traditional linear droop control is important for micro-grids in order to realize the plug and play characteristics. According to the defects of traditional linear droop control technology applied in DGs dealing with a large load demand fluctuation with the result of a larger frequency deviation problem, a new droop control method was presented. The new droop curve design is based on secondary frequency operations of synchronous generator trajectory, and the total frequency range was divided into segments, which consist of qualified and unqualified frequency segments by only primary frequency control. Through segmented design, the traditional primary frequency droop control is still used in the qualified frequency segment to keep the advantage of droop control. While in the unqualified frequency segment, the mathematical mapping function is built to project from the defect zone controlled by primary frequency operation to the aim projection zone controlled by secondary frequency operation. The mapping function builds the negative feedback relation between the source output power and frequency deviation to implement the automatic operation of secondary control. By means of the location set of projection points and the known droop coefficient value, the new nominal operation point can be located, which can be changed according to the load demand fluctuation in real time. The test in MATLAB/Simulink environments was carried out, and the results verified the validity of the auto-secondary control strategy, which deals with large load demand.
\end{abstract}

Keywords-microgrid; droop control; secondary automatic frequency control; mapping function; segment design

\section{INTRODUCTION}

Micro-grids play an important role in smart distribution networks via a flexible transition between grid-connected and -islanded modes as in [1-3]. The peer-to-peer control strategy provides the inherent advantage of seamless mode switching within micro-grids as in [4-11]. Linear droop control technology in peer-to-peer strategy for inverters can be consistent in grid-connected mode or islanded mode, potentially sharing load power automatically. One of the shortcomings of linear droop frequency control strategy, also called primary control, is the deviation from normal frequency magnitude in the face of large load power variations, which result in power quality problems. Secondary control strategy via moving the nominal output power value in synchronous generator operations can bring the unqualified frequency back to the qualified range. In the multi-inverters operation, a similar method is applied to adjust frequency. However, the realization procedure depends on the upper central command to determine the amount of the nominal output power value, or the location of the new nominal output point. The above method is not suitable to the application of the 'Plug and Play' characteristics in a micro-grid. Another method of adding an integral action to the droop controller improves the frequency deviation as was presented in [12-13], but this is not suitable in an islanded mode. The authors in [14-15] improve the above method in islanded mode by increasing more integral loops; however, more integral loops will weaken the response speed of controller. A novel distributed hierarchical structure is presented by basic communication in the island micro-grid [16] to improve the frequency deviation. Though the above method slightly depends on communication technology, the fault in communication affects the island operation. What's more, the addition of integral loop will make the source output much more power to make the frequency back the nominal value when faced with any load demand situation, which will make the heavy burden to the sources or generators. And the not frequency deviation regulation is not helpful to stable share of load demand between multi-inverter sources or multi-generators, which may be harmful to the stabilization of operation. Therefore, we implement a valuable method for improving frequency control based on the proportional element with the result of proper frequency deviation or sometimes no deviation. 
In this paper, a method with the automatic secondary frequency control function without the dependence of communication is presented to improve the large deviation problem that arises due to linear droop control in micro-grid inverters. The total frequency range is divided into segments, consisting of qualified and unqualified frequency segments only by primary frequency control. Through segmented design, the traditional primary frequency droop control is still used in the qualified frequency segment in order to keep the advantage of droop control. While in the unqualified frequency segment, the mathematical mapping function is built to project from the defect zone controlled by primary frequency operation to the aim projection zone controlled by secondary frequency operation. The mapping function builds the negative feedback relation between the source output power and the frequency deviation to implement the automatic operation of secondary control. By means of the location set of projection point and the known droop coefficient value, the new nominal operation point can be located, which can be changed according to the load demand fluctuation in real time.

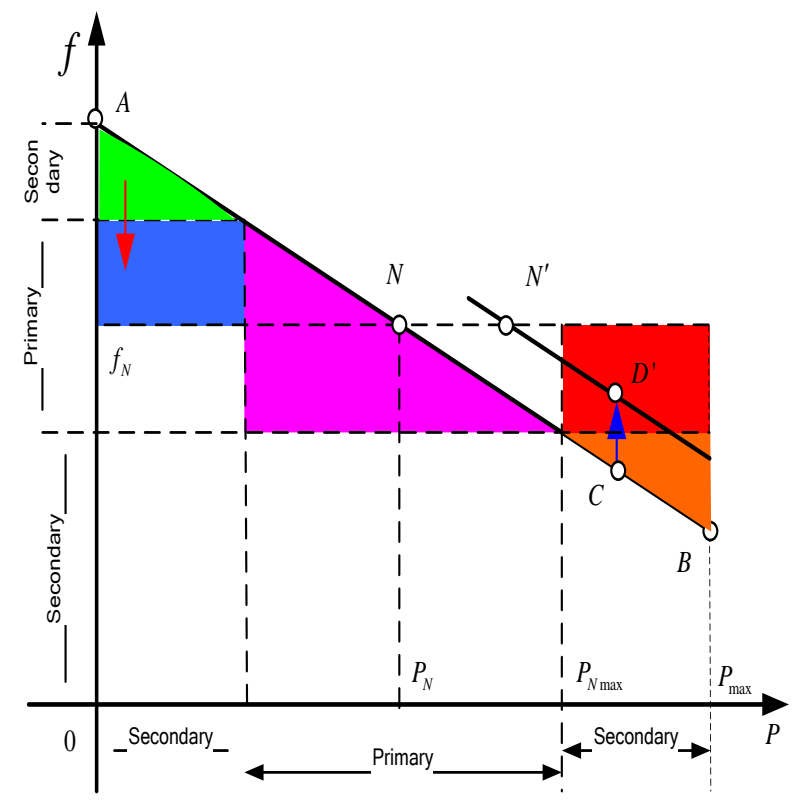

Figure 1. Generator primary and secondary frequency control segments diagram

\section{MicRo-GRID INVERTER DroOP CONTROL WITH}

\section{SECONDARY FREQUENCY CONTROL CHARACTERISTICS}

The analog of droop control characteristics in inverters was originally derived from the power output characteristics of synchronous generators. In normal operations, the frequency adjustments according to the size of load magnitude and cycle variations in synchronous generator operation actions can be divided into two cases: primary droop control action and secondary droop control action.

\section{A. Conception of Automatic Secondary Frequency Control}

According to the defects of traditional linear droop control technology applied in DGs in deal with a large load demand fluctuation with the result of larger frequency deviation problem, a new droop control conception was presented. In the concept, the droop curve design is based on secondary frequency operations trajectory of synchronous generator, and the total frequency range was divided into segments, which consist of primary frequency control segments and secondary frequency control segments. The former segment corresponds to the suitable frequency deviation and small magnitude variation of source output power. The segment is usually symmetrical with the nominal operating point. The latter segments correspond to the larger frequency variation and larger output power range beyond the ability of the only primary frequency control and usually located in the two zones respectively close to the output power upper and lower limits. The three triangle segments filled with different color can be found in Fig. 2. As can be seen, the pink triangle area denotes the primary frequency control zone, where the load demand can be satisfied by the only primary droop control of inverter. And the nominal operation point $\mathrm{N}$ is located in the above area, whose projection values to horizontal axis and to vertical axis are $P_{N}$ and $f_{N}$, respectively. The orange and green triangle areas are frequency defect zones with only primary control, which denote the too large frequency deviation areas resulted due to the only primary control facing the large load demand.

Through segmented design, the traditional primary frequency droop control is still used in the primary frequency segment to maintain the advantages of droop control. While in the secondary frequency segment, the mathematical mapping function is built to map from the frequency defect zone controlled by primary adjustment to the aim projection zone controlled by secondary frequency operation. In Fig. 2, the rectangle areas filled with red and blue are the aim projection zones where the frequency is located in the qualified range; the triangle areas filled with orange and green are the defect zone where the frequency is located in the unqualified range; the arrow denotes the mapping direction. The establishment of mapping relation is helpful to realize the aim of secondary frequency automatic control in inverters and to build the negative feedback relation between the source output power and the frequency deviation. The negative feedback relation make the inverters obtain the automatic share characteristics of load demand as same as the primary droop control without the help of communication. By means of the location set of the projection point and the known droop coefficient value, the new nominal operation point can be located, which can change according to the load demand fluctuation in real time. The projection point and the new nominal operation point are both located at the same droop curve. After relocating to the position of new nominal operation point, the new operation point will slightly move along the above droop curve facing anymore small load demand variation. The aim of all designs is to make the frequency fluctuate in the 
suitable ranges faced with the large load demand and make the control technology maintain the attributes of droop characteristics inherent to the automatic share of load demand among multi-inverters.

The task is to build the mapping function between the defect areas and the aim areas. The mapping direction is shown by the blue and red arrows in Fig. 1. The key problem in the secondary frequency control realization is the location of the projection point, which can be obtained by the linear or nonlinear map functions demonstrated in Fig. 1. No matter which function is selected, the new nominal operation point will be determined. If we want to obtain optimal operation, we need to continue designing the droop coefficients and map functions among multi-inverters.

\section{B. Design of the Mapping Function}

The design of the mapping function (mapping from the defect area [the orange or green triangle-area] to the aim area [the red or blue rectangle area]) is related directly to the projection point location, which determines the position of the new operation point and then the new nominal power output value. Because the practical load demand changes anytime, the characteristics are always variable. Therefore, the exact load model is impossible to build and the exact characteristic curve is impossible to obtain. The exact intersection point between the generator output power curve and the load demand curve cannot be determined; this is also referred to the stable operation point. But the approximate characteristics curve according to the relation of traditional load power and frequency can be deduced. The load vs frequency curve can be treated as a line with the positive slope. So the area surrounded by the source output power character curve and the load demand curve may be the projected aim zone. Due to the above analysis, any position in the aim area may be the new stable operation point; therefore, the mapping function can be built to fit many types.

To analyze the design of the mapping function directly in the diagram method, the area including the red aim area of Fig. 1 is enlarged, which is demonstrated in Fig. 2. The zone between the power $\mathrm{P}_{\mathrm{N}}$ and $\mathrm{P}_{\mathrm{Nmax}}$ is regarded as the primary control zone (only by primary frequency regulation), and the zone between the power $\mathrm{P}_{\mathrm{Nmax}}$ and $\mathrm{P}_{\max }$ is regarded as secondary control zone (mainly by means of secondary frequency regulation, if needed, with the addition of primary frequency regulation), as can be seen in Fig. 2. Due to the positive slope characteristics of load curve, the part of the red rectangle area can be regarded as the real aim area. Theoretically, any point in the red rectangle area may be regarded as the aim projection point, and thus any linear or nonlinear curve located at the above red zone can be built as the mathematical model of the mapping function. In order to simplify the mapping process, the heavy curve FA in Fig. 2 can be used to demonstrate as examples. And with the help of the source droop coefficients, the new nominal operation point position N' can be obtained. Also, the mathematical model of line FA can be built, and the new operation point is mapped into line FA. Due the characteristics of line FA, the new operation point position for the load demand and the new nominal point position for the source are overlapped.

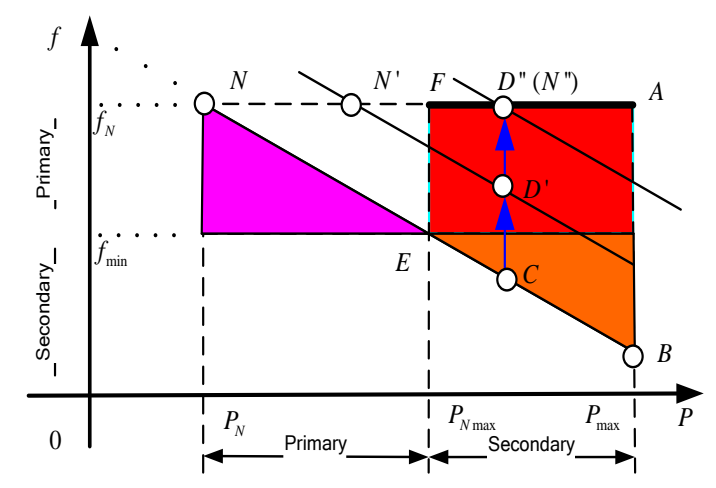

Figure 2. S-Shaped Droop Curve and Ratio Curve (Prim-Con: Primary Control; Secon-Con: Secondary Control)

Both line EA and line FA are artificially set. The effect of two kinds of sets will be analyzed. With the new operation point moving along line EA or FA to point A, the tendency is that the location of new nominal operation point is closer to the power output upper limit with the increment of load demand. The above tendency is consistent with practical operation conditions, but the increment amplitude is different in the two situations. The same operation point in the defect area, denoted as C, will be mapped seperately to the operation points D' and D' in the aim area while the nominal operation points, denoted as $\mathrm{N}^{\prime}$ and N', corresponding to the above points are different. The mapping point D' and the new nominal point N"' in line FA are the identical point. Due to the negative slope characteristics of source droop curve, the active power output of point $\mathrm{N}^{\text {" ' is }}$ far closer to the upper limit than that of point N'. Then the new operation point D', could possibly make the system frequency deviation zero. Meanwhile, the new operation point D' could make the system frequency deviation less than the original frequency deviation.

\section{Modeling of the Typical Mapping Function}

In order to determine the exact mapping function description, the model of the mapping lines FA, need be built in order to complete the later simulation process. The line FA denotes a zero slope condition. The two conditions are depicted below.

The model of line FA, the so-called zero slope model, can be deducted as:

$$
f_{\text {map }}=f_{N} \quad\left(P_{N \max }<P<P_{\max }\right)
$$

Based on the above formula, $\mathrm{P}_{\text {Nnew }}$ can be obtained as:

$$
P_{\text {Nnew }}=P_{N^{\prime \prime}}=P
$$

According to the comparison result of the new nominal output power value separately deduced from the above two functions, the new nominal power in the zero slope function is always bigger than the corresponding value in positive slope function due to the $\mathrm{k}_{\mathrm{p}}$ value with the negative sign. The 
above conclusion means that the source with a zero slope function always outputs more power than the source with a positive slope function, and then less deviation to the nominal frequency $\mathrm{f}_{\mathrm{N} .}$.

\section{Procedure of the Secondary Control Process}

The realization of automatic secondary control in multiinverters is based on the set of the frequency upper and lower limits, and the new nominal output power value is refreshed until the above set of frequency limit is broken through. The whole implementation process is designed based on the procedure of secondary frequency regulation (secondary control) in the synchronous machine, which consists of two parts: one being the secondary control to deal with the larger load demand, and the other being the primary control to deal with the small load demand or the latter load fluctuation after the secondary control. This procedure is illustrated in Fig. 3.

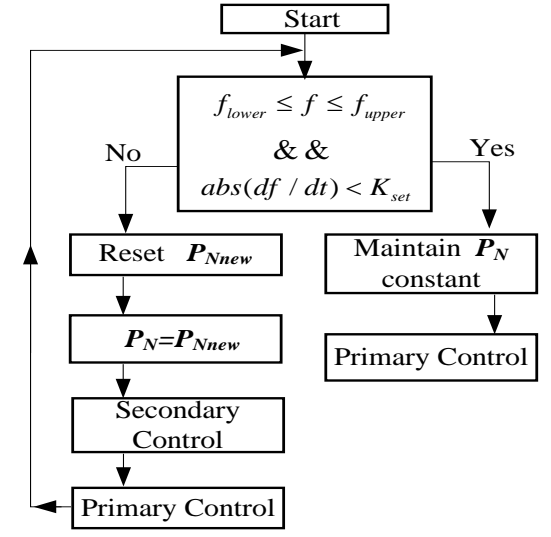

Figure 3. The secondary control procedure

In the above figure, if the frequency amplitude cannot meet with the regular range limits, the procedure will transfer to a loop. Meanwhile, the frequency fluctuation dynamic will affect the convergence speed and the duration period of fluctuation. So the gradient of frequency vs. time is included as the common criteria if transferring into the above loop or not. Then the coordination between the range limit and the gradient should be researched. If the absolute value of frequency gradient is large, the range limits should be narrow to remain some margin for the frequency adjustment, and vice versa.

According to the circulation procedure, it can be deduced that the operation number of circulations in practical conditions depends on the amplitude of unbalanced power between the source output and the load demand, as well as the number of inverters participating with the secondary control. The less is the unbalance power, the less is the circulation number. The more is the number of inverters participating in the secondary control, the less is the circulation number, and then the less is the number of frequency fluctuation. Obviously, faced with the same amount of unbalanced power, the multi-inverters, compared with the single inverter, have more powerful abilities and better adjustment effects. The practical operation condition can be consistent with the above analysis.

\section{IMPLEMENTATION OF THE S-SHAPED DROOP CHARACTERISTIC IN INVERTER CONTROL}

The linear droop control structure is comprised of a power controller and of current/voltage double-control loops, shown in Fig.4.

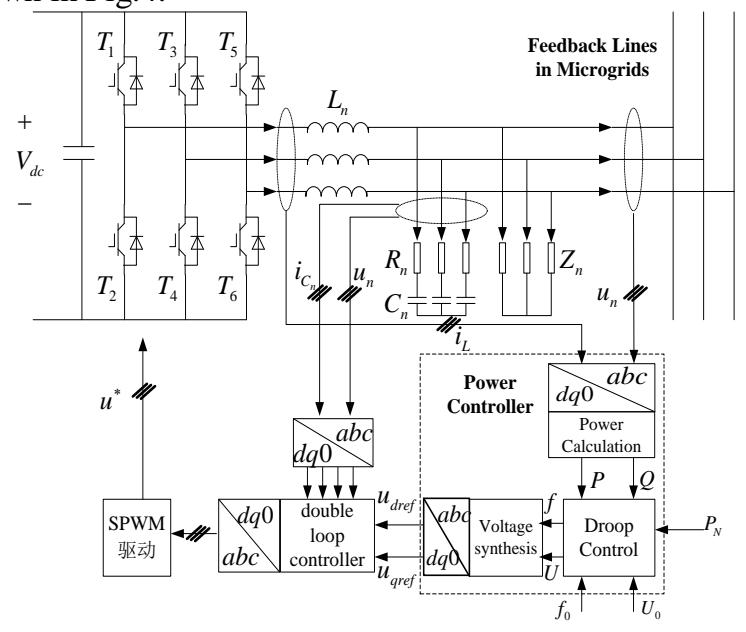

Figure 4. Droop control constitute diagram

The power controller consists of three parts: power calculation modules, droop control modules, and voltage synthesis modules, each with its specific internal constitution (Fig.4). The power calculation module collects the load voltage and current via sensors in order to calculate the DG instantaneous power output and then obtain the average power $\mathrm{P}$ and $\mathrm{Q}$ via low-pass filtering. According to outputs P and Q, the frequency and amplitude of the output voltage command value of $f$, $U$ will be obtained by the droop control where DG active power $\mathrm{P}$ and reactive power $\mathrm{Q}$ must meet the two conditions $0 \leq \mathrm{P} \leq \mathrm{P}_{\max }$ and $\mathrm{Q}_{\min } \leq \mathrm{Q} \leq$ $\mathrm{Q}_{\max }$. Voltage synthesis controller uses frequency $\mathrm{f}$ and voltage $U$ to generate a three-phase symmetrical voltage reference $\mathrm{u}_{\mathrm{ref}}$, and obtain $\mathrm{u}_{\mathrm{dref}}$, $\mathrm{u}_{\mathrm{qref}}$ by $\mathrm{dq}$ transformation, which acts as the input voltage and current to the double loop controller. The output is the controllable sinusoidal modulation signal $u$, which is input into the SPWM module.

During normal operations, the system frequency always lies within the regular range, which is to say the realization depends on the above control structure. When there is a sharp increment or decrement in load demand, the system frequency will deviate to the defect area, which means that the frequency broke through the lower or upper limits. Then the automatic secondary control action will be activated and the new nominal power output will be produced according to the value provided by the mapping function and will be maintained by the later load demand. Consequently, the maintenance of the new nominal output power value is fundamental to the secondary control process. The series of modifications will be carried out by the given nominal output power value in the droop control structure. The automatic secondary control will change the given nominal output power value facing the large load demand and will maintain the new modified value in the later process until the frequency limits are broken. 
In the Matlab /Simulink environment, the replacement of the old nominal power and maintenance of the new nominal power are too complicated to be calculated by means of the MATLAB program and port functions of Simulink. Hence, it is important to composite the current modules to realize the above function. Here it presents a feedback structure with the help of a logic-relational symbol, the closed loop. The structure cannot influence the nominal output value in the normal operation, and meanwhile it is responsible to realize the production of the new nominal output value via the superposition of the old nominal value.

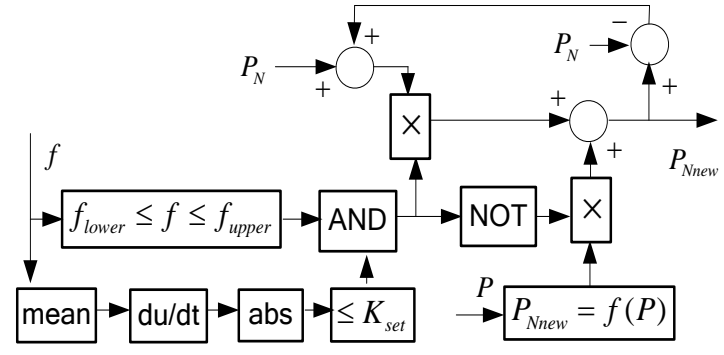

Figure 5. The new nominal power replacement and maintenance procedure

In Fig.5, the frequency sample is the real time frequency value, which can reflect the practical dynamics; and the mean module is used to avoid the rapid frequency fluctuation and the interference of noise value into the normal frequency sample.

\section{Simulation AND ANAlysis}

In order to verify the feasibility of the automatic secondary control applied in the micro-grid inverter control, the simulation is carried out in MATLAB/Simulink environments. The test micro-grid circuit is powered by two inverter sources, as shown in Fig.6. To compare the control effect between the automatic secondary droop control and the linear droop control, the simulation tests are divided into two groups: the test group and the contrast group. The two inverters in the test group are both controlled by the secondary control. In the contrast group, the inverters are both controlled by a linear droop.

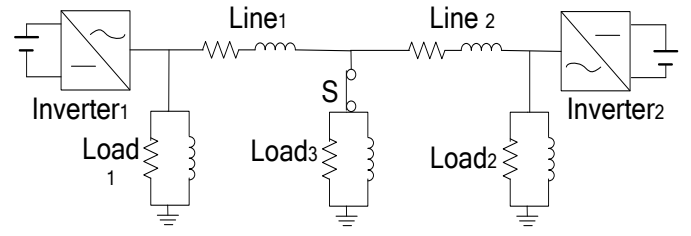

Figure 6. Micro-grid powered by two inverters diagram

The simulation set is as follows. The total duration length of the simulation is $0.5 \mathrm{~s}$; the initial state of the switch denoted by $\mathrm{S}$ is a closed set; the sampling interval is $5 \mathrm{e}-5 \mathrm{~s}$. The physical parameters of the two inverters are identical. The load parameters will select the maximum possible value in order to test the automatic secondary control effect. Here the regular frequency range and the system parameters are set as shown in Table I.
TABLE I. THE PARAMETERS OF SYSTEM AND SiMULATION

\begin{tabular}{|c|l|}
\hline Sources & $\mathrm{U}_{\mathrm{dc}}=800 \mathrm{~V}, \mathrm{P}_{\mathrm{N}}=50 \mathrm{~kW}, \mathrm{f}_{\mathrm{N}}=50 \mathrm{~Hz}, \mathrm{U}_{\mathrm{N}}=380 \mathrm{~V} ;$ \\
\hline Line impedance & $\mathrm{Z}_{\text {line1 }}=\mathrm{Z}_{\text {line } 2}=0.06+\mathrm{j} 0.1885 \Omega ;$ \\
\hline Frequency & $\mathrm{f}_{\text {upper }}=50.5 \mathrm{~Hz} ; \mathrm{f}_{\text {lower }}=49.5 \mathrm{~Hz} ;$ \\
\hline Power limits & $\mathrm{P}_{\mathrm{Nmax}}=100 \mathrm{~kW} ; \mathrm{P}_{\text {max }}=200 \mathrm{~kW}$ \\
\hline Droop control & $k_{p}=-1 \mathrm{e}-5 ; k_{u}=-3 \mathrm{e}-4$ \\
\hline Maximum load & $\mathrm{Load}_{1}=\mathrm{Load}_{2}=40+\mathrm{j} 20 \mathrm{kVA} ; \mathrm{Load}_{3}=60+\mathrm{j} 30 \mathrm{kVA}$ \\
\hline
\end{tabular}

Based on the above data in Table I, the new nominal output power in the zero slope mapping function can also be obtained.

$$
P_{\text {Nnew }}=P
$$

The zero slope mapping function has some merits, and the merits can be confirmed based on the curves in Fig. 7. The meaning of the symbols in the figure is the same as the symbols in Fig. 7.

From Fig.7, it appears that there is no difference in the active powers and reactive powers sharing of load demand, wherein shares are distributed equally.
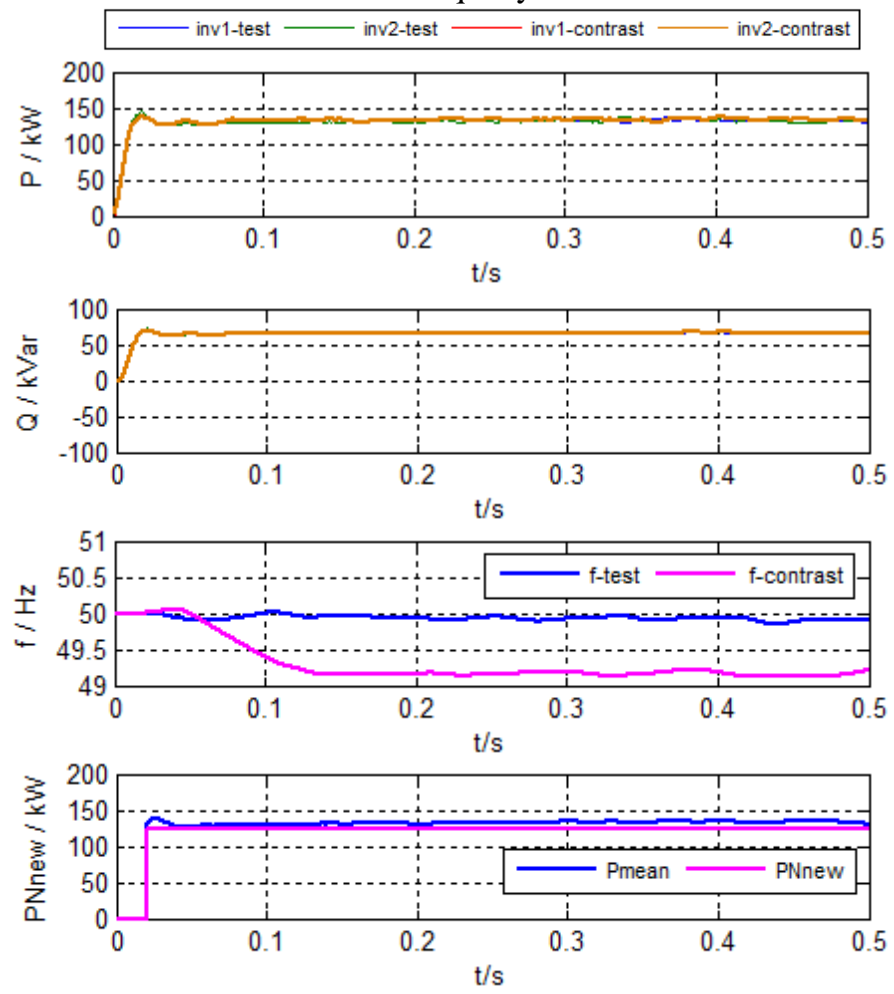

Figure 7. Simulation based on the zero slope mapping function

In the system frequency diagram, the frequency with secondary control reaches the stable frequency at approximate $50 \mathrm{~Hz}$, which means there is a no-deviating regulation. The ability of using only a primary control still locates the frequency at the defect zone. In the last diagram, the new nominal output power value is close to the power 
mean value, meaning there is a small imbalance of power between the source and the load. After the adjustment of the new nominal output power, there is slight primary control in the operation.

\section{CONCLUSION}

In this paper, frequency deviation problems existing in linear droop control are improved by means of automatic secondary droop control strategies, which can fit the output characteristics of the synchronous output power exactly. First, the frequency range is divided into two kinds of areas by only primary control: frequency qualified area and frequency unqualified area. By means of the mapping function, the operation point located in the unqualified area will be projected to the qualified area of frequency, and then the new nominal output power value is determined. After the adjustment of the new nominal power value, the simulation results confirm the validity of the present method.

\section{REFERENCES}

[1] Lasseter R.H. “Microgrids”, in Proc. 2001 Power Engineering Society Winter Meeting. pp.146-149

[2] Lasseter, R.H. and Paigi, P.. "Microgrid: a conceptual solution", in Proc. 2004 Power Electronics Specialists Conf, .pp. 4285 - 4290

[3] S. Chowdhury, S.P. Chowdhury and P. Crossley. Microgrids and Active Distribution Networks. London: The Institution of Engineering and Technology, pp. 251-258, 2009

[4] Alaa Mohda, Egon Ortjohanna, and Danny Mortonb, et al. "Review of control techniques for inverters parallel operationz". Electric Power Systems Research, vol 80, pp. 1477-1487, 2010

[5] J. Guerrero, J. Vasquez, J. Matas, M. Castilla, and L. García de Vicuña, "Control strategy for flexible microgrid based on parallel lineinteractive UPS systems," IEEE Trans. Ind. Electron., vol. 56, no. 3, pp. 726-736, Mar. 2009.

[6] S. V. Iyer, M. N. Belur, and M. C. Chandorkar, "A generalized computational method to determine stability of a multi-inverter microgrid,” IEEE Trans. Power Electron., vol. 25, no. 9, pp. 24202432, Sep. 2010.

[7] R. Majumder, B. Chaudhuri, A. Ghosh, G. Ledwich, and F. Zare, "Improvement of stability and load sharing in an autonomous microgrid using supplementary droop control loop,” IEEE Trans. Power Syst., vol. 25, no. 2, pp. 796-808, May 2010.

[8] M. Chandorkar, D. Divan, and R. Adapa, "Control of parallel connected inverters in standalone AC supply systems,” IEEE Trans. Ind. Appl., vol. 29, no. 1, pp. 136-143, Jan./Feb. 1993.

[9] J. M. Guerrero, J. C. V'asquez, J. Matas, M. Castilla, L. G. D. Vicu na, and M. Castilla, "Hierarchical control of droop-controlled $\mathrm{AC}$ and DC microgrids-A general approach toward standardization,” IEEE Trans.Ind. Electron., vol. 58, no. 1, pp. 158172, Jan. 2011

[10] Y. Li, D. Vilathgamuwa, and P. C. Loh, "Design, analysis, and realtime testing of a controller for multibus microgrid system," IEEE Trans. Power Electron., vol. 19, no. 5, pp. 1195-1204, Sep. 2004.

[11] F. Katiraei, M. R. Iravani, and P. W. Lehn, "Microgrid autonomous operation during and subsequent to islanding process," IEEE Trans. Power Del., vol. 20, no. 1, pp. 248-257, Jan. 2005

[12] Guo Tian-Yong, Zhao Geng-Shen, Zhao Yao, et al. "Modeling and simulation of microgrid system based on wind-solar hybrid". Power System Protection and Control, vol. 38, pp.104-106, 2010

[13] R. Majumder, B. Chaudhuri, A. Ghosh, G. Ledwich, and F. Zare. Improvement of stability and load sharing in an autonomous microgrid using supplementary droop control loop, IEEE Trans. Power Syst., 2010, 25( 2): 796-808.

[14] M. Marwali, J.-W. Jung, and A. Keyhani, "Control of distributed generation systems-Part II: Load sharing control,” IEEE Trans. Power Electron., vol. 19, no. 6, pp. 1551-1561, Nov. 2004.

[15] Qing-Chang Zhong. "Robust Droop Controller for Accurate Proportiona Load Sharing Among Inverters Operated in Parallel". IEEE Transactions on Industrial Electronics, vol. 60, pp. 1281-1290, April, 2013.

[16] John W. Simpson-Porco, Florian Dörfler, and Francesco Bullo "Synchronization and power sharing for droop-controlled inverters in islanded microgrids”. Automatica, vol. 49, pp. 2603-2611, June, 2013. 